\title{
Electrophysiologic Evaluation of Diffuse Axonal Injury after Traumatic Brain Injury
}

Yuko Urakami*

Department of Diagnosis and Treatment, National Rehabilitation Center for Persons with Disabilities, Tokorozawa Saitama 359-8555, Japan

\begin{abstract}
Background: Electroencephalography may provide useful information about consciousness and cognitive processing in patients who have traumatic brain injury. Sleep disturbance after traumatic brain injury may impair cognitive function and affect rehabilitation.

Methods: In 15 patients who had chronic diffuse axonal injury after traumatic brain injury, electroencephalography and neuropsychologic tests were performed. In 8 patients who had subacute and chronic diffuse axonal injury and 7 healthy control subjects, electroencephalography, magnetoencephalography, and neuropsychologic tests were performed to evaluate sleep spindles and cognitive function.

Results: In the chronic stage of diffuse axonal injury, the mean peak frequency of alpha activity was significantly slower in patients who had abnormal than normal electroencephalography. The mean peak frequency of fast spindles, amplitude, and cortical activation source strength in precentral and postcentral regions were significantly slower in patients who had subacute diffuse axonal injury than healthy participants, and these parameters increased from the subacute to the chronic stage of diffuse axonal injury. After neurocognitive rehabilitation, cognitive functions were improved in all patients.
\end{abstract}

Conclusion: Alpha activity reflects the severity of disturbed consciousness in the acute stage after traumatic brain injury. Spindles will be an indicator of the recovery of consciousness in the chronic stage. Electroencephalographic makers may be useful in the diagnosis and prognosis of traumatic brain injury.

Keywords: Electroencephalography; Magnetoencephalography; Neuropsychologic; Cognition; Sleep spindles; Alpha activity

Abbreviations: DAI: Diffuse Axonal Injury; EEG: Electroencephalography; F: Female; GCS: Glasgow Coma Scale; GOS: Glasgow Outcome Scale; HDS-R: Hasegawa Dementia Scale Revised; M: Male; MEG: Magnetoencephalography; MMSE : Mini Mental State Examination; NA : Not available; PIQ : Performance Intelligence Quotient; TIQ: Total Intelligence Quotient; TBI: Traumatic Brain Injury; VIQ : Verbal Intelligence Quotient; WAIS-R : Wechsler Adult Intelligence Scale Revised; WISC-R: Wechsler Intelligence Scale for Children Revised

\section{Introduction}

In patients who have traumatic brain injury (TBI), a diagnosis and treatment are essential for acute patient care and long-term rehabilitation. Many problems may occur after TBI, including somatic problems (headache, fatigue, sexual dysfunction, and sleep disturbance), cognitive dysfunction (impairment of memory, attention, and information processing capacity) [1], mood changes (depression, aggression, emotional liability, and anxiety), and post-traumatic personality changes (self-centered behavior, reduced social awareness, and disinhibited emotions resulting from frontal and temporal lobe damage) [2,3].

Electroencephalography (EEG) has been evaluated for the clinical assessment of consciousness to support the diagnosis and prognosis $[4,5]$. Electrical activity of brain tissue may have good prognostic value after brain injury. When performed from 15 days to 4 years after injury, EEG may provide an objective and quantitative measure of the severity of brain injury [6]. Furthermore, EEG can detect early seizure activity, and provide information about sleep patterns during polysomnography. Sleep disturbances are common after TBI, and may include, insomnia, hypersomnia, and altered sleep-wake cycles [7-9]. However, there are no reproducible findings about specific changes in sleep quality and sleep architecture measured during polysomnography. Changes in sleep architecture after TBI are inconsistent, because the neural mechanisms contributing to sleep disturbances are multifaceted. The degree of damage to sleep-wake regulating centers such as the ascending reticular formation and associated pathways or neurotransmitter systems affect sleep disturbances $[10,11]$. Anxiety and depression frequently occur after TBI, and increased depression is associated with decreased sleep quality $[12,13]$.

Proper diagnosis and effective treatment for sleep disorders after TBI, such as post-traumatic sleep-wake disturbances, possibly will improve the quality of sleep and improve the learning of motor tasks and cognition during sleep. Therefore, this study performed a study to evaluate the diagnosis utility of EEG in patients who had chronic diffuse axonal injury (DAI). In addition, simultaneously performed EEG and magnetoencephalography(MEG) in patients who had subacute to chronic DAI to evaluate the possibility of spindles as a biomarker.

\section{Materials and Methods}

\section{Electroencephalography in the diagnosis of chronic diffuse} axonal injury

The present study was approved by the ethics committee of National

*Corresponding author: Yuko Urakami, Department of Diagnosis and Treatment National Rehabilitation Center for Persons with Disabilities, Tokorozawa Saitama 359-8555 Japan; Tel: +81.42.995.3100; Fax: +81.42.995.0355; E-mail: Urakamiyuko@rehab.go.jp

Received June 24, 2013; Accepted July 22, 2013; Published July 30, 2013

Citation: Urakami Y (2013) Electrophysiologic Evaluation of Diffuse Axonal Injury after Traumatic Brain Injury. J Neurol Neurophysiol 4: 157. doi:10.4172/21559562.1000157

Copyright: @ 2013 Urakami Y. This is an open-access article distributed unde the terms of the Creative Commons Attribution License, which permits unrestricted use, distribution, and reproduction in any medium, provided the original author and source are credited. 
Citation: Urakami Y (2013) Electrophysiologic Evaluation of Diffuse Axonal Injury after Traumatic Brain Injury. J Neurol Neurophysiol 4: 157. doi:10.4172/2155-9562.1000157

Rehabilitation Center for Persons with Disabilities. The study of EEG included 15 patients (13 male and 2 female: mean age, 23) who were diagnosed with acute DAI and who participated in our cognitive inpatient rehabilitation program. Coma duration was $>6$ hours, and magnetic resonance imaging showed little evidence of focal cerebral lesions.

The EEG recordings were performed in the chronic stage (mean time after injury $332 \pm 550 \mathrm{~d}$ ), with 19 electrodes according to the international 10-20 system of electrode placement, with a linkedear reference (bandpass filter, 0.3 to $120 \mathrm{~Hz}$, time constant, 0.3). The EEG data were evaluated initially by visual inspection, and fast Fourier transform was computed to calculate the power spectrum of alpha activity for each interval of $0.25 \mathrm{~Hz}$ with a prism power spectrum (GE Marqettes, Tokyo, Japan). Ten epochs of stable alpha activity were summed and averaged. Cognitive functions were evaluated with neuropsychologic tests including Wechsler Adult Intelligence Scale- Revised (WAIS-R), which included Total Intelligence Quotient (TIQ), Verbal Intelligence Quotient (VIQ), and Performance Intelligence Quotient (PIQ); Mini-mental scale examination (MMSE); and Hasegawa Dementia Scale Revised (HDS-R).

\section{Sleep spindles of patients who had subacute to chronic diffuse axonal injury}

The study of sleep spindles included patients who had with DAI ( 7 male and 1 female; age range 21 to $37 \mathrm{y}$; mean age $26 \mathrm{y}$ ) and 7 agematched healthy volunteer control subjects ( 5 male and 2 female; age range, 24 to- $32 \mathrm{y}$; mean age; $28 \mathrm{y}$ ).

Simultaneous EEG and MEG recordings were performed twice in the patients who had DAI: (1) during the sub-acute stage (mean time after injury $80 \mathrm{~d}$ ), upon admission to the hospital for cognitive neurologic rehabilitation, and (2) during the chronic stage (mean time after injury, $151 \mathrm{~d}$ ), upon completion of inpatient rehabilitation. In control subjects, the simultaneous EEG and MEG recordings were performed once.
Data were recorded during natural sleep (approximately $30 \mathrm{~min}$ ) for each participant. The EEG recordings were made with a 60 -channel electrode cap (Elekta- Neuromag, Finland) that was placed on the scalp according to the international 10-10 system of electrode placement. Magnetic signals were recorded from 204 planar gradiometers (Vectorview, Elekta- Neuromag, Finland) with a band-pass of 0.03 to $300 \mathrm{~Hz}$ for both the EEG and MEG signals. The sampling rate for digital conversion was $1001 \mathrm{~Hz}$. For analysis, 10-stable fast spindles were selected by frequency and distribution. The Wechsler Adult Intelligence Scale III (WAIS-III) was used to evaluate cognitive functions.

\section{Data analysis}

Comparisons were performed with $t$ test. Statistical significance was defined by $\mathrm{P}<.05$.

\section{Results}

\section{Electroencephalography in the diagnosis of chronic diffuse axonal injury}

All patients were diagnosed as DAI and were evaluated with EEG (Table1). In the chronic stage, 9 patients $(60 \%)$ had abnormal EEG findings (Table2), including paroxysmal discharge noted in 4 patients (26\%), centrotemporal spikes, 5 to $6 \mathrm{~Hz}$ small spikes and wave phantom. Cognitive function which was measured with WAIS-R, WAIS-III, MMSE, or HDS-R was similar in patients who had normal or abnormal EEG (Table 2). The mean peak frequency of alpha activity was significantly slower in patients who had abnormal than normal EEG (Table 2).

\section{Sleep spindles of patients who had subacute to chronic diffuse axonal injury}

Eight patients who had DAI and who were evaluated for sleep

\begin{tabular}{|c|c|c|c|c|c|c|c|c|c|c|}
\hline \multirow[t]{2}{*}{ Patient No. } & \multirow[t]{2}{*}{ Age (y) } & \multirow[t]{2}{*}{ Sex } & \multicolumn{2}{|c|}{ Acute stage } & \multirow{2}{*}{$\begin{array}{c}\text { Chronic stage } \\
\text { Time After Injury When } \\
\text { EEG Performed (d) }\end{array}$} & \multirow{2}{*}{$\begin{array}{c}\text { Cognitive Function } \\
\text { MMSE }\end{array}$} & \multirow[b]{2}{*}{ HDS-R } & & \multirow[b]{2}{*}{ WAIS-R } & \multirow[b]{3}{*}{ PIQ } \\
\hline & & & GCS & $\begin{array}{c}\text { Coma } \\
\text { Duration (d) }\end{array}$ & & & & & & \\
\hline & & & & & & & & TIQ & VIQ & \\
\hline 1 & 20 & $M$ & 7 & 4 & 150 & 29 & 29 & 82 & 91 & 75 \\
\hline $2 \dagger$ & 32 & $M$ & 7 & 7 & 120 & 23 & 22 & 48 & 60 & 45 \\
\hline $3 \dagger$ & 22 & $M$ & 7 & 30 & 210 & 27 & 26 & 96 & NA & NA \\
\hline 4 & 14 & M & 7 & 7 & 90 & 30 & 26 & 83 & 91 & 78 \\
\hline 5 & 21 & $M$ & 5 & 15 & 90 & 23 & 19 & 58 & 66 & 60 \\
\hline 6 & 17 & $\mathrm{M}$ & 7 & 3 & 90 & 15 & 18 & 50 & 64 & 45 \\
\hline 7 & 27 & M & 7 & 100 & 300 & Tanaka-Binet test: 2 years & 10 & Scale out & & \\
\hline 8 & 23 & M & 7 & 10 & 150 & 27 & 28 & 64 & 71 & 67 \\
\hline 9 & 27 & M & 7 & 180 & 300 & Tanaka-Binet test: 2 years & Scale out & Scale out & & \\
\hline 10 & 20 & M & 7 & 40 & 300 & 29 & 29 & 50 & 71 & 46 \\
\hline 11 & 17 & M & 7 & 30 & 240 & Tanaka-Binet test: 2 years & 8 & Scale out & & \\
\hline 12 & 38 & $\mathrm{~F}$ & 7 & 14 & 180 & 23 & 28 & 84 & 82 & 89 \\
\hline $13 \dagger$ & 25 & $\mathrm{~F}$ & 9 & 80 & 2370 & 23 & 28 & 64 & 88 & 45 \\
\hline $14 \dagger$ & 26 & M & 7 & 60 & 300 & 19 & 19 & 58 & 52 & 58 \\
\hline $15 \dagger$ & 17 & $M$ & 6 & 7 & 90 & 19 & 28 & 78 & 84 & 75 \\
\hline Mean \pm SD & $23 \pm 6$ & & $6.9 \pm 0.8$ & $39 \pm 49$ & $332 \pm 570$ & $24 \pm 5$ & $23 \pm 7$ & $68 \pm 16$ & $75 \pm 13$ & $62 \pm 16$ \\
\hline
\end{tabular}

${ }^{*} \mathrm{~N}=15$ patients (13 male and 2 female). All patients had diffuse axonal injury. Abbreviations: EEG, electroencephalography; F, female; GCS, Glasgow Coma Scale; HDS-R, Hasegawa Dementia Scale Revised; M, male; MMSE, Mini Mental State Examination; NA, not available; PIQ, Performance Intelligence Quotient; TIQ, Total Intelligence Quotient; VIQ, Verbal Intelligence Quotient; WAIS-R, Wechsler Adult Intelligence Scale Revised; WISC-R, Wechsler Intelligence Scale for Children Revised; Scale out; could not perform neuropsychologic test due to severe mental dysfunction.

† Patient had subarachnoid hemorrhage (total, 5 patients)

Table 1: Clinical and cognitive characteristics of patients who had diffuse axonal injury after traumatic brain injury*. 
Citation: Urakami Y (2013) Electrophysiologic Evaluation of Diffuse Axonal Injury after Traumatic Brain Injury. J Neurol Neurophysiol 4: 157. doi:10.4172/2155-9562.1000157

spindles were young adult males who had moderate to severe injury (Table 3). In both healthy subjects and patients who had DAI, the centers of cortical activation of the 2 types of spindles (fast spindles and slow spindles) were located in 4 areas: the precentral areas of the right and left posterior frontal cortex and the postcentral areas of the right and left posterior parietal cortex. The precentral activation areas for fast spindles were located in the primary motor cortex and for slow spindles were located in the anterior frontal cortex, including the supplementary motor cortex. The postcentral activation areas for both types of spindles were located in the posterior parietal cortex. The postcentral activation areas included the parietal cortex of the primary sensorimotor areas and the posterior parietal areas. In healthy subjects, the cortical activation strength was similar between the four areas, including precentral and postcentral areas of both hemisperes (Table4). The mean peak frequency of fast spindles, amplitude, and cortical activation source strength in precentral and postcentral regions were significantly slower in patients who had DAI, the mean peak frequency of fast spindles, amplitude, and cortical activation source strength in precentral and postcentral regions were significantly faster in the chronic than subacute stage (Table 4).The mean peak frequency of fast spindles, amplitude, and cortical activation source strength in

\begin{tabular}{|c|c|c|c|c|}
\hline \multicolumn{2}{|c|}{ Characteristic } & Normal EEG & Abnormal EEG & $P \leq \dagger$ \\
\hline \multicolumn{2}{|c|}{ No. patients } & 6 & 9 & \\
\hline \multicolumn{2}{|c|}{ Coma duration (d) } & $11 \pm 10$ & $58 \pm 59$ & .01 \\
\hline \multicolumn{2}{|c|}{ Time after injury (d) } & $125 \pm 44$ & $470 \pm 676$ & .01 \\
\hline \multicolumn{5}{|l|}{ WAIS-R } \\
\hline & $T I Q$ & 64.2 & $66.3 \ddagger$ & NS \\
\hline & $\mathrm{VIQ}$ & 74.0 & 74.6 & NS \\
\hline & $\mathrm{PIQ}$ & 60.6 & 63.3 & NS \\
\hline MMSE & & 24.5 & 25.0 & NS \\
\hline HDS-R & & 24.0 & 26.6 & NS \\
\hline \multicolumn{2}{|c|}{ Alpha activity peak frequency $(\mathrm{Hz})$} & $10.1 \pm 0.6$ & $9 \pm 1$ & .05 \\
\hline
\end{tabular}

${ }^{*} \mathrm{~N}=15$ patients. Abbreviations: EEG, electroencephalography; HDS-R, Hasegawa Dementia Scale Revised; MMSE, Mini Mental State Examination; PIQ, Performance Intelligence Quotient; TIQ, Total Intelligence Quotient; VIQ, Verbal Intelligence Quotient; WAIS-R, Wechsler Adult Intelligence Scale Revised

†NS, not significant $(P>.05)$

$\ddagger$ Mean for 6 patients; 3 other patients could not perform neuropsychologic tests due to severe intelligence disturbance.

Table 2: Relation between electroencephalography during the chronic stage and cognitive function in patients who had diffuse axonal injury after traumatic brain injury*. precentral and postcentral regions were similar between patients who had DAI( chronic stage) and healthy participants (Table 4). There were no differences in mean peak frequency of fast spindles, amplitude, and cortical activation source strength in precentral and postcentral regions between hemispheres in healthy participants or patients who had DAI (subacute or chronic stages).

All 8 patients who had DAI had cognitive dysfunction, including memory disturbances and attention deficit. After neurocognitive rehabilitation, cognitive function improved in all patients, especially significant improvement in the WAIS-III subset scores for verbal intelligence quotient, performance intelligence quotient, total intelligence quotient, perceptual organization, and working memory (Table 5). All patients had favorable clinical outcome at 1 year after injury, with Glasgow Outcome Scale scores from 4 to 5. Some cognitive dysfunctions persisted after rehabilitation, but all patients returned home or resumed work.

\section{Discussion}

In the present study, the spontaneous EEG activity and cognitive function were evaluated after DAI. The EEG activity may be a marker of the severity of consciousness disturbance (acute stage) and recovery (chronic stage) after DAI. Although quantitative analysis was required for this study, alpha activity and spindles may be distinguished by visual inspection during typical clinical EEG studies [14]. Therefore, the present findings may be applicable to clinical practice. In patients who had TBI, it is important to consider the combined interaction and coordination of disturbed brain processes that are represented by the EEG, Glasgow Coma Scale, loss of consciousness, and neuropsychologic tests [15].

Previous quantitative EEG studies have attempted to develop an objective and quantitative measure of the severity of brain injury by using EEG obtained from 15 days to 4 years after injury $[4,6,16]$. The multivariate clinical assessment is a global variable that considers several neurophysiologic processes that are affected by TBI, especially those involving the frontal and temporal lobes [6].

The parieto-occipital alpha rhythm was attenuated by eye opening, visual stimuli, and by increased attentiveness [17]. These findings contributed to the hypothesis that oscillations may function as an 'idling' rhythm that characterizes an alert, yet inactive brain state. Recently published data have confirmed that alpha oscillations are strengthened by internal tasks, such as mental calculation and mental imagery [18-20]. The alpha band amplitude is increased during

\begin{tabular}{|c|c|c|c|c|c|c|c|}
\hline \multirow[t]{2}{*}{ Patient No. } & \multirow[t]{2}{*}{ Age (y) } & \multirow[t]{2}{*}{ GCS } & \multirow[t]{2}{*}{ Severity† } & \multirow[t]{2}{*}{ Time in Intensive Care Unit (d) } & \multirow[t]{2}{*}{ GOS } & \multicolumn{2}{|l|}{ Time After Injury (d) } \\
\hline & & & & & & 1st Examination & 2nd Examination \\
\hline 1 & 22 & 7 & Severe & 10 & 5 & 92 & 163 \\
\hline 2 & 21 & 7 & Severe & 14 & 5 & 51 & 124 \\
\hline 3 & 24 & 10 & Moderate & 1 & 5 & 39 & 98 \\
\hline 4 & 37 & 9 & Moderate & 1 & 5 & 62 & 132 \\
\hline 5 & 24 & 7 & Severe & 20 & 5 & 72 & 142 \\
\hline 6 & 34 & 7 & Severe & 7 & 5 & 56 & 123 \\
\hline 7 & 22 & 7 & Severe & 5 & 4 & 82 & 182 \\
\hline 8 & 24 & 6 & Severe & 4 & 4 & 182 & 243 \\
\hline Mean \pm SD & $26 \pm 6$ & $8 \pm 1$ & & $8 \pm 7$ & $4.8 \pm 0.5$ & $80 \pm 45$ & $151 \pm 45$ \\
\hline
\end{tabular}

${ }^{*} \mathrm{~N}=8$ patients ( 7 male and 1 female; patient no. 2 was female). Cause of injury was a traffic accident in 7 patients and a fall in 1 patient (patient no. 8 ). All patients had favorable outcome at 1 year. Examination included electroencephalography and magnetoencephalography. Abbreviations: GCS, Glasgow Coma Scale; GOS, Glasgow Outcome Scale

†Severity was defined by the GCS score, posttraumatic amnesia, and duration of loss of consciousness when the first physician arrived Table 3: Characteristics of patients who had evaluation of sleep spindles after diffuse axonal injury* 
Citation: Urakami Y (2013) Electrophysiologic Evaluation of Diffuse Axonal Injury after Traumatic Brain Injury. J Neurol Neurophysiol 4: 157. doi:10.4172/2155-9562.1000157

Page 4 of 6

\begin{tabular}{|c|c|c|c|c|c|c|c|}
\hline \multicolumn{2}{|l|}{ Characteristic } & & \multirow[t]{2}{*}{ Healthy Subjects } & \multicolumn{4}{|c|}{ Patients Who Had Diffuse Axonal Injury } \\
\hline & & & & Subacute & $P \leq \dagger$ & Chronic & $P \leq \ddagger$ \\
\hline \multicolumn{8}{|c|}{ Peak frequency in central areas } \\
\hline & EEG $(\mathrm{Hz})$ & & & & & & \\
\hline & & Left & $13.9 \pm 0.2$ & $13.4 \pm 0.1^{*}$ & .05 & $13.8 \pm 0.2$ & .05 \\
\hline & & Right & $13.9 \pm 0.2$ & $13.5 \pm 0.1^{*}$ & .05 & $13.9 \pm 0.2$ & .05 \\
\hline & MEG $(\mathrm{Hz})$ & & & & & & \\
\hline & & Left & $13.7 \pm 0.2$ & $13.3 \pm 0.1^{*}$ & .05 & $13.7 \pm 0.2$ & .05 \\
\hline & & Right & $13.7 \pm 0.1$ & $13.3 \pm 0.1^{*}$ & .05 & $13.8 \pm 0.2$ & .05 \\
\hline \multicolumn{8}{|c|}{ Amplitude in central areas } \\
\hline & EEG $(\mu \mathrm{V})$ & & & & & & \\
\hline & & Left & $29 \pm 10$ & $28 \pm 10^{*}$ & .05 & $29 \pm 9$ & .05 \\
\hline & & Right & $30 \pm 9$ & $29.5 \pm 0.1^{*}$ & .05 & $31 \pm 8$ & .05 \\
\hline & $\mathrm{MEG}(\mathrm{fT} / \mathrm{cm})$ & & & & & & \\
\hline & & Left & $84 \pm 29$ & $68 \pm 32^{*}$ & .05 & $87 \pm 38$ & .05 \\
\hline & & Right & $90 \pm 34$ & $70 \pm 31^{*}$ & .05 & $101 \pm 18$ & .05 \\
\hline \multicolumn{8}{|c|}{ Cortical activation source strength on MEG } \\
\hline & Precentral & & & & & & \\
\hline & & Left & $39 \pm 19$ & $22 \pm 12^{*}$ & .05 & $37 \pm 16$ & .05 \\
\hline & & Right & $39 \pm 20$ & $17 \pm 16^{*}$ & .05 & $40 \pm 20$ & .05 \\
\hline & Postcentral & & & & & & \\
\hline & & Left & $38 \pm 21$ & $20 \pm 18^{*}$ & .05 & $38 \pm 20$ & .05 \\
\hline & & Right & $35 \pm 17$ & $21 \pm 19^{*}$ & .05 & $36 \pm 18$ & .05 \\
\hline
\end{tabular}

${ }^{*} \mathrm{~N}=7$ healthy subjects and 8 patients who had diffuse axonal injury. Data reported as mean \pm SD. There were no differences in mean peak frequency of fast spindles amplitude, and cortical activation source strength in precentral and postcentral regions between patients who had diffuse axonal injury (chronic stage) and healthy participants. Abbreviations: EEG, electroencephalography; MEG, magnetoencephalography

†Difference between healthy subjects and patients who had diffuse axonal injury (subacute stage).

$\ddagger$ Patients who had diffuse axonal injury; difference between subacute and chronic stages.

Table 4: Characteristics of fast spindles in healthy subjects and patients who had diffuse axonal injury after traumatic brain inury*

\begin{tabular}{|c|c|c|c|c|c|c|c|c|}
\hline Patient No. & Stage & WAIS-III & & & & & & \\
\hline & & VIQ & PIQ & TIQ & Verbal comprehension & Perceptual organization & Working memory & Processing speed \\
\hline \multirow[t]{2}{*}{1} & Subacute & 76 & 63 & 65 & NA & NA & NA & NA \\
\hline & Chronic & $102 \dagger$ & $96 \dagger$ & $98 \dagger$ & NA & NA & NA & NA \\
\hline \multirow[t]{2}{*}{2} & Subacute & 56 & 50 & 49 & 54 & 55 & 54 & 50 \\
\hline & Chronic & $112 \dagger$ & $98 \dagger$ & $101 \dagger$ & 98 & $102 \dagger$ & $98 \dagger$ & 97 \\
\hline \multirow[t]{2}{*}{3} & Subacute & 96 & 102 & 98 & 86 & 106 & 103 & 81 \\
\hline & Chronic & $110 \dagger$ & $98+$ & $102 \dagger$ & 98 & $110 \dagger$ & $111 \dagger$ & 92 \\
\hline \multirow[t]{2}{*}{4} & Subacute & 88 & 84 & 85 & 90 & 83 & 100 & 84 \\
\hline & Chronic & $111 \dagger$ & $97 \dagger$ & $113 \dagger$ & 102 & $110 \dagger$ & 92 & $102 \dagger$ \\
\hline \multirow[t]{2}{*}{5} & Subacute & 59 & 58 & 55 & 61 & 61 & 60 & 57 \\
\hline & Chronic & 86 & $72 \dagger$ & 88 & 72 & $82 \dagger$ & $88 \dagger$ & 66 \\
\hline \multirow[t]{2}{*}{6} & Subacute & 64 & 74 & 65 & 56 & 66 & 62 & 63 \\
\hline & Chronic & $84 \dagger$ & $102 \dagger$ & $91 \dagger$ & 92 & $119 \dagger$ & $76 \dagger$ & 78 \\
\hline \multirow[t]{2}{*}{7} & Subacute & 50 & 53 & 103 & 86 & 112 & 111 & 60 \\
\hline & Chronic & $90 \dagger$ & $103+$ & $95 \dagger$ & 86 & $112 \dagger$ & $111 \dagger$ & 60 \\
\hline \multirow[t]{2}{*}{8} & Subacute & 114 & 90 & 104 & 109 & 101 & 111 & 81 \\
\hline & Chronic & 120 & 94 & 110 & 110 & $118 \dagger$ & 110 & 92 \\
\hline
\end{tabular}

*N = 8 patients. Abbreviations: PIQ, Performance Intelligence Quotient; TIQ, Total Intelligence Quotient; VIQ, Verbal Intelligence Quotient; WAIS-III, Wechsler Adult Intelligence Scale III. NA; not available

†Difference between subacute and chronic stages: $P \leq .05$

Table 5: Cognitive function in patients who had traumatic brain injury*.

the period of retention of short-term- and working memory and is suppressed subsequently. A previous study suggested that these largeamplitude oscillations during memory retention may inhibit the retrieval of memorized items, and this may be reflected in subsequent amplitude suppression. The present study showed longer coma duration in the acute stage, and slower frequency of alpha activity in the chronic stage of DAI. Further study may clarify whether alpha oscillations are associated with idling, inhibition, attention, or binding within the Global neural networks after brain damage that may cause consciousness disturbance in patients who have DAI.

In patients who have coma, the arousal system of the brain is deregulated because of diffuse brain or focal brainstem damage. In 
coma the EEG may have various patterns of activity including, a generalized slowing in the delta or theta range, alpha- coma, spindlecoma, burst- suppression, and epileptiform activity [21-23]. The normal sleep-wake cycle is disrupted or completely absent in coma. However, the coma tracing resemble normal wakefulness or normal sleep [24]. Spindle coma, characterized by the occurrence of spindles in comatose patients, may be caused by central nervous system trauma, infection, or metabolic encephalopathy. Abnormal spindle formations occur because of midbrain involvement with sparing of thalamic structures [25]. In patients who have supratentorial lesions, spindle coma suggests the presence of intact cortical function and good prognosis [26,27]. Spindle- coma is considered as a benign form of coma, with EEG reactivity to stimuli that may herald a favorable outcome [28,29]. In comatose patients, spindles are best demonstrated during first few days after trauma. In a previous study, spindle activity was observed in $91 \%$ patients who had posttraumatic coma, and $30 \%$ patients developed prolonged coma [29].

In the acute stage of coma, spindles may have prognostic value. Symmetric occurrence of spindles may be associated with a good prognosis, and asymmetric or decreased spindles may be associated with a poor prognosis [26]. In patients who have chronic coma, recovery may occur over many months or years. Focal injury patterns in the central thalamus are associated with coma, vegetative state, and a minimally conscious state. Medial components of the posterior intralaminar region of the central thalamus are involved in spindle generation. Therefore, the recovery of consciousness may be strongly associated with the recovery of sleep spindles after brain injury [30,31].

Sleep disorders are common after the acute phase of TBI. Cognitive dysfunctions may be caused by sleep disturbances and may worsen the impairment in patients who have TBI. Sleep disturbances may include hypersomnia, insomnia, altered sleep-wake cycles, periodic limb movements in sleep, and disorders of rapid eye movement sleep. Breathing disorders of rapid eye movement sleep such as obstructive sleep apnea or central apnea. In patients who have obstructive sleep apnea have greater impairment of neurocognitive function, especially memory and sustained attention, than patients who do not have disordered breathing during sleep.

In patients who have TBI, findings of sleep architecture are inconsistent and may include; no changes, increased slow waves, decreased rapid eye movement sleep, increased rapid eye movement sleep during the second half of the night, no change in rapid eye movement sleep, or decreased onset latency of rapid eye movement sleep. Sleep-wake regulating centers and associated pathways are damaged in TBI, and these damages will be the cause of disturbances of sleep architecture. Sleep disturbances contribute to fatigue, which may be associated with mental slowness and, slowed information processing [32]. Sleep disturbances also contribute to anxiety and depression. Patients with TBI may have disrupted circadian regulation of melatonin synthesis, including lower levels of melatonin production during the evening [12]. Reduced sleep quality induces depression. Early diagnosis and treatment with modafinil, melatonin, and light therapy may improve alertness and mood. It also is important to support lifestyle modifications, and facilitate daytime activities [33].

In summary, the present study showed that electrophysiologic markers such as alpha activity and sleep spindles may be useful in the diagnosis and prognosis of TBI. The multivariate clinical assessment is a global variable that may combine several neurophysiologic that are needed to access prognosis.
Proper diagnosis and effective treatment of sleep disorders after TBI may potentially improve the quality of sleep, the off-line learning of motor tasks and cognition during sleep, alertness, mood, and rehabilitation.

\section{Acknowledgement}

This article was supported by Grants-in Aid for Scientific Research 23500473 (Japan Society for the Promotion of Science).

\section{References}

1. Schacter DL, Crovitz HF (1977) Memory function after closed head injury: a review of the quantitative research. Cortex 13: 150-176.

2. Rimel RW, Giordani B, Barth JT, Boll TJ, Jane JA (1981) Disability caused by minor head injury. Neurosurgery 9: 221-228.

3. Kwentus JA, Hart RP, Peck ET, Kornstein S (1985) Psychiatric complications of closed head trauma. Psychosomatics 26: 8-17.

4. Thatcher RW, Walker RA, Gerson I, Geisler FH (1989) EEG discriminant analyses of mild head trauma. Electroencephalogr Clin Neurophysiol 73: 94 106.

5. Dockree PM, Robertson IH (2011) Electrophysiological markers of cognitive deficits in traumatic brain injury: a review. Int J Psychophysiol 82: 53-60.

6. Thatcher RW, North DM, Curtin RT, Walker RA, Biver CJ, et al. (2001) An EEG severity index of traumatic brain injury. J Neuropsychiatry Clin Neurosci 13 $77-87$

7. Castriotta RJ, Wilde MC, Lai JM, Atanasov S, Masel BE, et al. (2007) Prevalence and consequences of sleep disorders in traumatic brain injury. Clin Sleep Med 3: 349-356.

8. Orff HJ, Ayalon L, Drummond SP (2009) Traumatic brain injury and sleep disturbance: a review of current research. J Head Trauma Rehabil 24: 155-165.

9. Castriotta RJ, Murthy JN (2011) Sleep disorders in patients with traumatic brain injury: a review. CNS Drugs 25: 175-185.

10. Parcell DL, Ponsford JL, Redman JR, Rajaratnam SM (2008) Poor sleep quality and changes in objectively recorded sleep after traumatic brain injury: a preliminary study. Arch Phys Med Rehabil 89: 843-850.

11. Frieboes RM, Müller U, Murck H, von Cramon DY, Holsboer F, et al. (1999) Nocturnal hormone secretion and the sleep EEG in patients several months after traumatic brain injury. J Neuropsychiatry Clin Neurosci 11: 354-360.

12. Shekleton JA, Parcell DL, Redman JR, Phipps-Nelson J, Ponsford JL, et al. (2010) Sleep disturbance and melatonin levels following traumatic brain injury Neurology 74: 1732-1738.

13. Rao V, Spiro J, Vaishnavi S, Rastogi P, Mielke M, et al. (2008) Prevalence and types of sleep disturbances acutely after traumatic brain injury. Brain Inj 22 381-386.

14. Hughes JR, John ER (1999) Conventional and quantitative electroencephalography in psychiatry. J Neuropsychiatry Clin Neurosci 11: 190-208.

15. Katz DI, Alexander MP (1994) Traumatic brain injury. Predicting course of recovery and outcome for patients admitted to rehabilitation. Arch Neurol 51: 661-670.

16. John ER, Prichep LS, Fridman J, Easton P (1988) Neurometrics: computerassisted differential diagnosis of brain dysfunctions. Science 239: 162-169.

17. Palva S, Palva JM (2007) New vistas for alpha-frequency band oscillations Trends Neurosci 30: 150-158.

18. Cooper NR, Burgess AP, Croft RJ, Gruzelier JH (2006) Investigating evoked and induced electroencephalogram activity in task-related alpha power increases during an internally directed attention task. Neuroreport 17: 205-208.

19. Jensen O, Gelfand J, Kounios J, Lisman JE (2002) Oscillations in the alpha band $(9-12 \mathrm{~Hz})$ increase with memory load during retention in a short-term memory task. Cereb Cortex 12: 877-882.

20. Sauseng P, Klimesch W, Doppelmayr M, Pecherstorfer T, Freunberger R, et al. (2005) EEG alpha synchronization and functional coupling during top-down processing in a working memory task. Hum Brain Mapp 26: 148-155. 
Citation: Urakami Y (2013) Electrophysiologic Evaluation of Diffuse Axonal Injury after Traumatic Brain Injury. J Neurol Neurophysiol 4: 157. doi:10.4172/2155-9562.1000157

21. Cologan V, Schabus M, Ledoux D, Moonen G, Maquet P, et al. (2010) Sleep in disorders of consciousness. Sleep Med Rev 14: 97-105.

22. LOEB C, POGGIO G (1953) Electroencephalograms in a case with pontomesencephalic haemorrhage. Electroencephalogr Clin Neurophysiol 5: 295296.

23. JASPER H, VAN BUREN J (1955) Interrelationship between cortex and subcortical structures: clinical electroencephalographic studies. Electroencephalogr Clin Neurophysiol Suppl Suppl.

24. Hughes JR, Cayaffa J, Leestma J (1976) Electro-clinical pathologic correlations in comatose patients.Clin Electroencephalogr 7: 13-30.

25. SIVERMAN D (1963) RETROSPECTIVE STUDY OF THE EEG IN COMA. Electroencephalogr Clin Neurophysiol 15: 486-503.

26. Rumpl E, Prugger M, Bauer G, Gerstenbrand F, Hackl JM, et al. (1983) Incidence and prognostic value of spindles in post-traumatic coma. Electroencephalogr Clin Neurophysiol 56: 420-429.

27. Britt CW Jr (1981) Nontraumatic "spindle coma": clinical, EEG, and prognostic features. Neurology 31: 393-397.
28. Britt CW Jr, Raso E, Gerson LP (1980) Spindle coma, secondary to primary traumatic midbrain hemorrhage. Electroencephalogr Clin Neurophysiol 49: 406-408.

29. Seet RC, Lim EC, Wilder-Smith EP (2005) Spindle coma from acute midbrain infarction. Neurology 64: 2159-2160.

30. Urakami Y, Ioannides AA, Kostopoulos GK (2012) Chapter 4. Sleep Spindles -As a biomarker of brain function and plasticity. Advances in Clinical Neurophysiology, Edited by Ihsan M. Ajeena. ISBN 978-953-51-0806-1.73-108.

31. Urakami Y (2012) Relationship between, sleep spindles and clinical recovery in patients with traumatic brain injury: a simultaneous EEG and MEG study. Clin EEG Neurosci 43: 39-47.

32. Ponsford JL, Ziino C, Parcell DL, Shekleton JA, Roper M, et al. (2012) Fatigue and sleep disturbance following traumatic brain injury--their nature, causes, and potential treatments. J Head Trauma Rehabil 27: 224-233.

33. Cicerone KD, Dahlberg C, Kalmar K, Langenbahn DM, Malec JF, et al. (2000) Evidence-based cognitive rehabilitation: recommendations for clinical practice. Arch Phys Med Rehabil 81: 1596-1615.

This article was originally published in a special issue, Traumatic Brain Injury: Diagnosis \& Treatment handled by Editor(s). Douglas Mckay Wallace, University of Miami, USA 